\title{
Topological insulator: a new quantized spin Hall resistance robust to dephasing
}

\author{
Hua Jiang ${ }^{1}$, Shuguang Cheng $^{1}$, Qing-feng Sun ${ }^{1, *}$, and X. C. Xie ${ }^{2,1}$ \\ ${ }^{1}$ Beijing National Lab for Condensed Matter Physics and Institute of Physics, \\ Chinese Academy of Sciences, Beijing 100190, China; \\ ${ }^{2}$ Department of Physics, Oklahoma State University, Stillwater, Oklahoma 74078
}

(Dated: August 4, 2021)

\begin{abstract}
The influence of dephasing on the quantum spin Hall effect (QSHE) is studied. In the absence of dephasing, the longitudinal resistance in a QSHE system exhibits the quantum plateaus. We find that these quantum plateaus are robust against the normal dephasing but fragile with the spin dephasing. Thus, these quantum plateaus only survive in mesoscopic samples. Moreover, the longitudinal resistance increases linearly with the sample length but is insensitive to the sample width. These characters are in excellent agreement with the recent experimental results [science 318, 766 (2007)]. In addition, we define a new spin Hall resistance that also exhibits quantum plateaus. In particular, these plateaus are robust against any type of dephasing and therefore, survive in macroscopic samples and better reflect the topological nature of QSHE.
\end{abstract}

PACS numbers: 73.43.-f.

Recently, the quantum spin Hall effect (QSHE), existed in a new quantum state of matter with a non-trivial topological property, has generated great interest. [1] QSHE occurs in the topological insulator with a bulk energy gap and two helical edge states crossing inside the gap. This energy-band structure guarantees that the carriers only flow along the boundary and that carriers with opposite spin-polarizations move in opposite directions on a given edge. The other key ingredient for QSHE is the presence of the spin-orbit interaction (SOI). When electrons move under an electric field, the SOI drives the electrons with opposite spins to deflect to the opposite transverse boundaries, and the special energy-band structure leads to the quantum spin Hall conductance. [1] The existence of QSHE was first proposed in a graphene film in which the SOI opens a bandgap around the Dirac-points and establishes the edge states. 2, 3]. Soon afterwards, QSHE was predicted to exist in some other two- or threedimensional systems. 4, 5, 6, 7] In particular, Bernevig et al. recently found that $\mathrm{CdTe} / \mathrm{HgTe} / \mathrm{CdTe}$ quantum well has an "inverted" type energy-band structure with proper well thicknesses [7] where QSHE naturally exists. Soon after this work, QSHE was successfully realized in an experiment [8, 9] in which a quantized longitudinal resistance plateau was observed when the sample's electron density was varied in the absence of a magnetic field. [8]

However, in the experiment of Ref.[8], the quantized longitudinal resistance plateaus could only emerge in mesoscopic samples. This character is very different from the regular quantum Hall effect (QHE). In QHE, the Hall resistance plateaus exist in macroscopic samples, robust against the impurity scattering as well as the inelastic (dephasing) scattering. This leads some to speculate that the inelastic scattering which induces phase relaxation, destroys the quantized plateaus in QSHE[1, 7], however, there has been no theoretical or experimental investigation thus far.

In this Letter, we study how QSHE is affected by de- phasing. We mainly focus on two questions: (i) How does dephasing affect the quantized longitudinal resistance plateau of QSHE samples as measured in the recent experiment [8]; (ii) Is there an observable physical quantity showing a quantized value in macroscopic samples, reflecting the topological nature of QSHE?

In a realistic sample, there are in general a number of possible dephasing processes, but these can be classified into two categories. In the first kind, the carriers lose only the phase memory while maintaining the spin memory, such as with the dephasing processes caused by the electron-electron interaction, the electron-phonon interaction, etc, these are named normal dephasing in this paper. In the second kind, the carriers lose both phase and spin memories, such as with the spin-flip dephasing processes caused by the magnetic impurities, the nuclear spins, etc, named spin dephasing. We consider a six-terminal device (shown in Fig.1a), as in the experimental set-up [8], and the dephasing processes are simulated by using the Büttiker's virtual probes. [10] By applying the Landauer-Büttiker formalism combined with the non-equilibrium Green function method [11, 12], the longitudinal resistance is calculated. The results show that the longitudinal resistance exhibits the quantum plateaus without dephasing or with the normal dephasing, but are then destroyed by the spin dephasing. Thus, these quantum plateaus are only observable in a mesoscopic sample in which the sample length is smaller than the spin-dephasing length. Our theoretical results provide a good understanding of the experimental findings of the dependence of the longitudinal resistance on temperature, sample length and sample width. More interestingly, we introduce a novel spin Hall resistance that also exhibits the quantized plateaus. In particular, these plateaus survive under both normal and spin dephasings, and are thus observable in macroscopic samples, similar to the conventional QHE.

In the tight-binding representation, the Hamiltonian of 
the six-terminal QSHE device can be written as 12, 13.

$$
\begin{aligned}
H= & -\left[\sum_{<\mathbf{i} \mathbf{j}>\sigma} t e^{i \eta(\sigma) \phi_{\mathbf{i} \mathbf{j}}} c_{\mathbf{i} \sigma}^{\dagger} c_{\mathbf{j} \sigma}+H . c .\right]+\left[\sum_{\mathbf{i} k \sigma} \epsilon_{k \sigma}\right. \\
& \left.a_{\mathbf{i} k \sigma}^{\dagger} a_{\mathbf{i} k \sigma}+\left(t_{k \sigma} a_{\mathbf{i} k \sigma}^{\dagger} c_{\mathbf{i} \sigma}+H . c .\right)\right]
\end{aligned}
$$

The first term describes the QSHE system including the central region and the six terminals. $c_{\mathbf{i} \sigma}^{\dagger}\left(c_{\mathbf{i} \sigma}\right)$ is the creation (annihilation) operator of an electron on the lattice site $\mathbf{i}$ with spin $\sigma, t=\hbar^{2} / 2 m^{*} a^{2}$ represents the nearest hopping matrix element with the lattice constant $a$. Due to the SOI, an extra spin-dependent phase $\eta(\sigma) \phi_{\mathbf{i j}}$ is added in the hopping element, with $\eta(\sigma)=1$ and -1 for $\sigma=\uparrow$ and $\downarrow$. 14] The summation of four $\phi_{\mathbf{i j}}$ along each unit satisfies $\sum_{\square} \phi_{\mathbf{i j}}=e B_{\text {eff }} a^{2} / \hbar$, with an effective magnetic field $B_{\text {eff }}$ coming from the SOI. The second term represents the Hamiltonian of virtual leads and their couplings to the central sites. Here we assume that the dephasing only occurs in the central region, and each site $\mathbf{i}$ in the central region is attached by a virtual lead. The size of the central region is $(L+2 M) \times W$ as shown in Fig.1a. In additional, if we take $\eta(\sigma)=1$ in Eq.(1), it describes a QHE system. 15]

Using multi-probe Landauer-Büttiker formula, the current in the lead-p (either real or virtual lead) with spin index $\sigma$ can be expressed as

$$
J_{p \sigma}=(e / \hbar) \sum_{q \neq p} T_{p q}^{\sigma}\left(V_{p \sigma}-V_{q \sigma}\right),
$$

where $V_{p \sigma}$ is the spin-dependent bias in the lead p. $T_{p q}^{\sigma}=$ $\operatorname{Tr}\left[\boldsymbol{\Gamma}_{\mathbf{p} \sigma} \mathbf{G}^{\mathbf{r}} \boldsymbol{\Gamma}_{\mathbf{q} \sigma} \mathbf{G}^{\mathbf{a}}\right]$ is the transmission coefficient from the lead-q to p with spin $\sigma$, where the linewidth functions $\boldsymbol{\Gamma}_{\mathbf{p} \sigma}=i\left[\boldsymbol{\Sigma}_{\mathbf{p} \sigma}^{\mathbf{r}}-\boldsymbol{\Sigma}_{\mathbf{p} \sigma}^{\mathbf{r}+}\right]$, the Green function $\mathbf{G}^{r}=\left[\mathbf{G}^{a}\right]^{\dagger}=$ $\left[E_{F} \mathbf{I}-\mathbf{H}_{c e n}-\sum_{p \sigma} \boldsymbol{\Sigma}_{p \sigma}^{r}\right]^{-1}, \mathbf{H}_{c e n}$ is the Hamiltonian in the central region, and $\boldsymbol{\Sigma}_{\mathbf{p}}^{\mathbf{r}}$ is the retarded self-energy due to the coupling to the lead-p. [12] For the real lead-p $(p=1,2,3,4,5,6)$, the self-energy $\boldsymbol{\Sigma}_{\mathbf{p}}^{\mathbf{r}}$ can be calculated numerically. For the virtual leads, $\boldsymbol{\Sigma}_{\mathbf{p}}^{\mathbf{r}}=-i \Gamma / 2$ and $\Gamma$ is the dephasing strength.

In our simulations, a small external bias is applied between the lead-1 and lead-4 with $V_{1 \uparrow}=V_{1 \downarrow}=-V_{4 \uparrow}=$ $-V_{4 \downarrow}=V$, which drives a current $I_{14}=I_{1}=-I_{4}$ flowing along the longitudinal direction. For normal dephasing, electrons only lose the phase memory while maintain the spin memory by going into and then coming back from the virtual leads. Thus, for each virtual lead$\mathbf{i}$ the currents have the constraint that $J_{\mathbf{i} \uparrow}=J_{\mathbf{i} \downarrow}=0$, and $V_{\mathbf{i} \uparrow}$ is usually not equal to $V_{\mathbf{i} \downarrow}$. But for spin dephasing, electrons can lose both phase and spin memories, so one has $V_{\mathbf{i} \uparrow}=V_{\mathbf{i} \downarrow}$ and $J_{\mathbf{i} \uparrow}+J_{\mathbf{i} \downarrow}=0$ for each virtual lead-i. In the recent experiment, [8] the four transverse real leads are the voltage probes, so $J_{p \uparrow}+J_{p \downarrow}=0$ and $V_{p \uparrow}=V_{p \downarrow} \equiv V_{p}$ for $p=2,3,5$, and 6. Combining Eq.(2) together with all boundary conditions for the real and virtual leads, the voltage $V_{p \sigma}$ and current $J_{p \sigma}$ in each real lead can be obtained. Then the longitudinal resistance

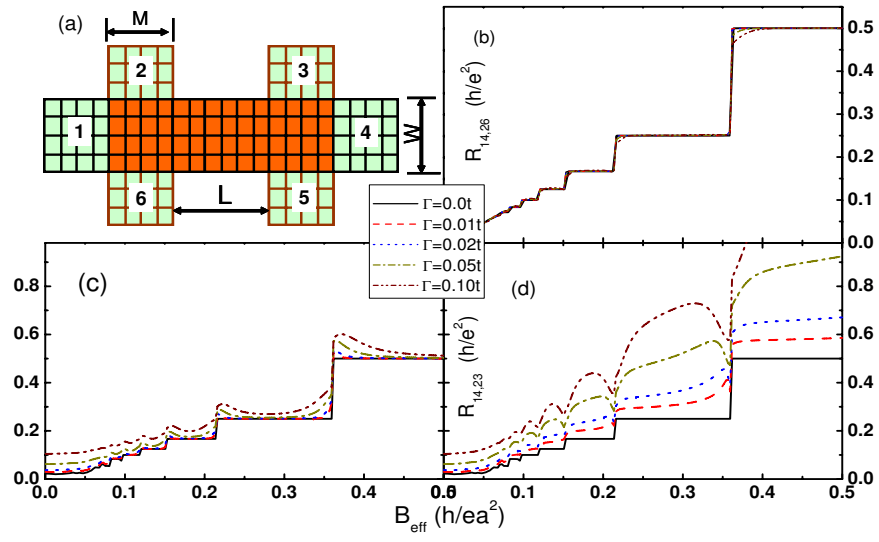

FIG. 1: (color online) (a) Schematic diagram for a sixterminal Hall bar sample, the gray (or red) area is the central region containing dephasing. (b) The Hall resistance $R_{14,26}$ vs. the magnetic field $B$ for different dephasing strengths $\Gamma$. (c) and (d) illustrate the longitudinal resistance $R_{14,23}$ vs $B_{\text {eff }}$ in the presence of normal and spin dephasings, respectively. The parameters are $M=24 a, L=32 a$ and $W=32 a$.

$R_{14,23} \equiv V_{23} / I_{14}=\left(V_{2}-V_{3}\right) /\left(I_{1 \uparrow}+I_{1 \downarrow}\right)$ and $I_{2 s} / I_{14}=$ $\left(I_{2 \uparrow}-I_{2 \downarrow}\right) / I_{14}$ can be calculated and will be presented next. Here $R_{14,23}$ is the measured quantity in the recent experiment. 8] In addition, we also consider the case in which the four transverse leads are taken as spin-bias probes with their currents $J_{p \uparrow}=J_{p \downarrow}=0$. In this case, we define a new spin Hall resistance $R_{s} \equiv\left(V_{i \uparrow}-V_{i \downarrow}\right) / I_{14}$ ( $i$ can be any transverse lead, for instance $i=2$ ), and its result will be shown in this study as well.

In the numerical calculations, we take the hopping matrix element $t=1$ as the energy unit. The Fermi energy is selected at $E_{F}=-3 t$ which is near the energy-band bottom $-4 t$. Since the flux in a unit lattice is $\phi=1$ when the efficient magnetic field $B_{\text {eff }}=h /\left(e a^{2}\right), h /\left(e a^{2}\right)$ was taken as the unit of $B_{e f f}$. The dephasing strength is described by the parameter $\Gamma$, which is directly related to the phase coherence length $L_{\phi}[15]$, an experimental observable parameter. Fig. $2 \mathrm{~b}$ and $2 \mathrm{c}$ show the relation of $L_{\phi}$ vs $\Gamma$. With increasing $\Gamma, L_{\phi}$ decreases rapidly and monotonically for either normal or spin dephasing. To test out our model, we first investigate the effect of dephasing on the integer QHE [i.e. $\eta(\sigma)=1$ in the Hamiltonian (1)]. As shown in Fig.1b, the quantized Hall resistance plateaus of $R_{14,26}$ in $\mathrm{QHE}$ is hardly affected by either dephasing, in agreement with previous experimental and theoretical results. 15]

Now, we present our numerical results of the dephasing effect on QSHE. Fig.1c and 1d show the longitudinal resistance $R_{14,23}$ versus $B_{\text {eff }}$ for the normal and spin dephasings with different dephasing strength $\Gamma$. In the absence of dephasing (solid lines), $R_{14,23}$ exhibits perfect quantum plateaus at $h / 2 \nu e^{2}(\nu=1,2,3, \ldots)$. Note that in the experiment of Ref. [8], only one plateau was observed 


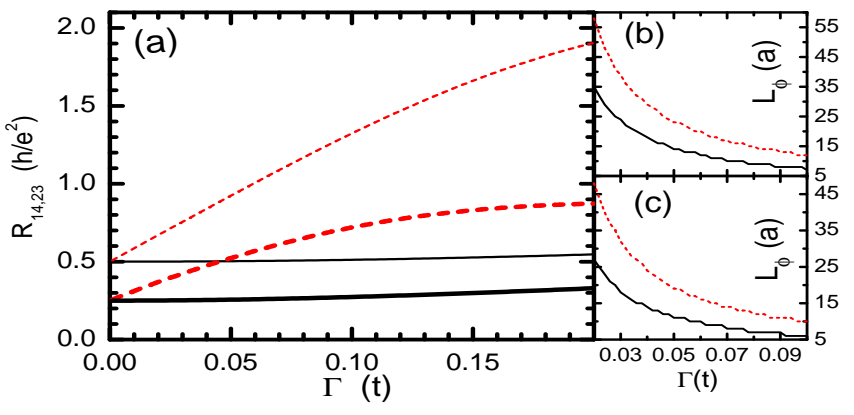

FIG. 2: (color online) (a) shows $R_{14,23}$ vs. $\Gamma$ with $L=32 a$, $W=32 a, M=24 a$, and $B_{\text {eff }}=0.5$ (thin curves) and 0.3 (thick curves). (b) and (c) show $L_{\phi}$ vs. $\Gamma$ at $B_{\text {eff }}=0.5$ (b) and 0.3 (c) with $W=32 a$. The solid and dotted curves in $(\mathrm{a}, \mathrm{b}, \mathrm{c})$ are for the normal and spin dephasings.

since only one edge channel is there for each spin component at a given edge. In our theoretical model of Eq.(1), one allows multi-channels. Thus, the experimental situation corresponds to the highest plateau at $h / 2 e^{2}$ with $\nu=1$ in our model. In the presence of dephasing, the quantum plateaus of $R_{14,23}$ behave quite differently depending on the type of dephasing. With normal dephasing, the plateau structure remains and $R_{14,23}$ changes only slightly in between the plateaus. For normal dephasing, temperature causes the dephasing broadening in $\Gamma$, thus, this shows that QSHE is insensitive to $T$ at low temperatures. However, from Fig.(1d), one sees that with spin dephasing, $R_{14,23}$ increases significantly even with small $\Gamma$.

Next, we investigate the effect of dephases in more detail. Fig.2a shows $R_{14,23}$ versus dephasing strength $\Gamma$ for fixed $B_{\text {eff }}=0.5$ and 0.3 , which are at the centers of the 1st and 2nd plateaus. For the normal dephasing, the increase of $R_{14,23}$ is extremely slow with increasing $\Gamma$. For example, at $\Gamma=0.2 t$, the sample size is about one order larger than the phase coherence length $L_{\phi}$, but $R_{14,23}$ is only increased by less $4 \%$. In contrast, for the spin dephasing, $R_{14,23}$ increases rapidly with increasing $\Gamma$. For example, even for a small $\Gamma=0.02 t$, in which the sample length is shorter than $L_{\phi}, R_{14,23}$ is increased by about $16 \%$. For normal dephasing, the carriers maintain their spin memories, and backscattering only occurs when a carrier is scattered from one boundary to the opposite one. So the backscattering is very weak except when the Fermi energy is near a Landau level center, and the quantum plateaus of $R_{14,23}$ can survive even with very large normal dephasing. But for the spin dephasing, the spin of a carrier can be flipped, and the backscattering occurs on each boundary. So the longitudinal resistance $R_{14,23}$ is strongly affected by the spin dephasing. In a real experimental sample, the spin dephasing always exists to some degree, due to magnetic impurities, nuclear spin fluctuations, etc., thus, the quantum plateaus of longitudinal resistance of QSHE only survive in mesoscopic

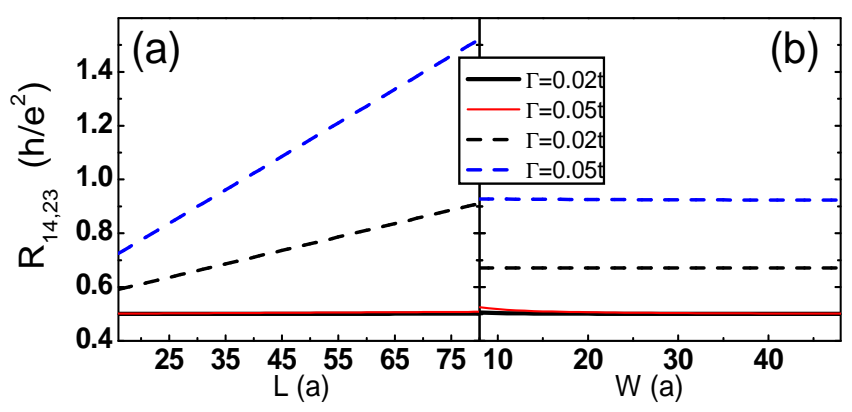

FIG. 3: (color online) (a) and (b) show $R_{14,23}$ vs. the sample length $L$ with $W=32 a$ (a) and the width $W$ (b) with $L=32 a$ (b), $M=24 a$, and $B_{\text {eff }}=0.5$. The solid and dash lines are for the normal and spin dephasings, respectively.

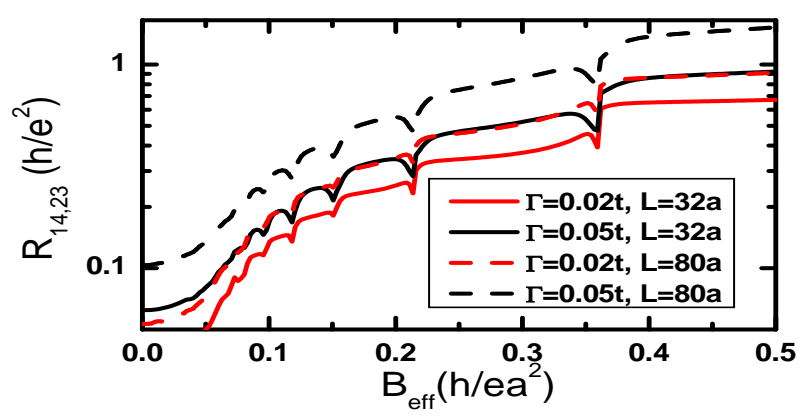

FIG. 4: (color online) $R_{14,23}$ vs. $B_{\text {eff }}$ for different sample lengths $L$ and spin-dephasing strengths $\Gamma$ with $W=32 a$ and $M=24 a$.

samples. This explains why the quantum plateau was not observed in samples with large lengths. [8]

In Fig. $3, R_{14,23}$ dependence on the system sizes is studied. For normal dephasing, the plateau of $R_{14,23}$ stays at the quantized value regardless of the sample length $L$ or the width $W$ since the backscattering is weak in all cases except for very small $W$. On the other hand, for spin dephasing, $R_{14,23}$ is almost independent of the width $W$ but is linearly increasing with the length $L$ since the backscattering is stronger with larger $L$. In Fig.4, we plot $R_{14,23}$ in logarithmic scale as done in the experimental figures. [8] Similar to the experimental plots, $R_{14,23}$ approximatively shows the plateau characteristics regardless of the dephasing strength $\Gamma$, although the plateau values may well exceed the idealized quantized-values of $h / 2 \nu e^{2}$ in the absence of spin dephasing. Combining all the results from Figs.1-4, we qualitatively explain the experimental findings on the behavior of the longitudinal resistance $R_{14,23}$ and its dependence on temperature, sample length and sample width. [8]

Up to now, we find that the quantum plateaus of longitudinal resistance $R_{14,23}$ survive only for mesoscopic samples due to spin dephasing. Whether there exists an observable physical quantity in macroscopic QSHE samples? Or more importantly, is there an observable quantity better reflect the topological nature of the QSHE 


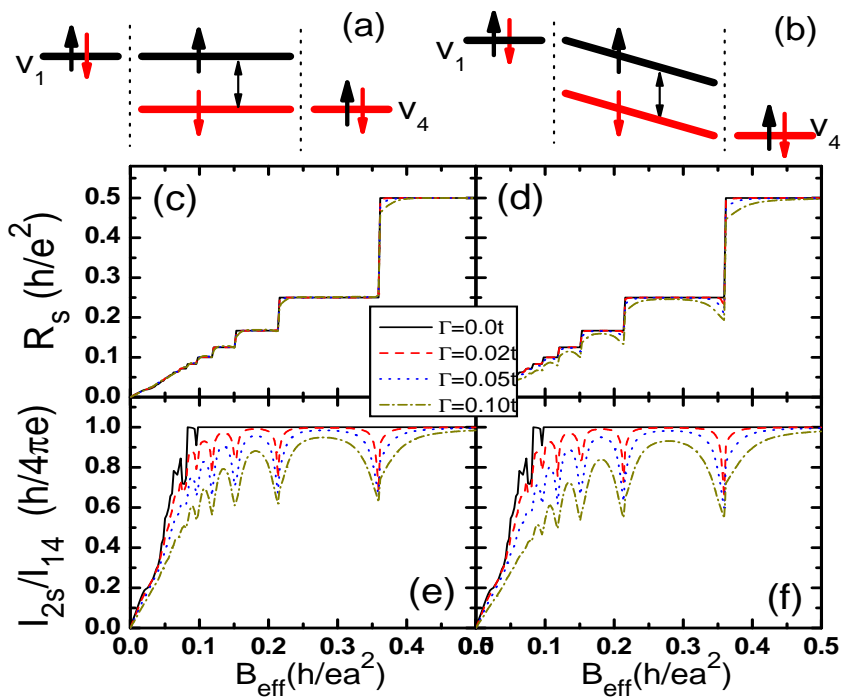

FIG. 5: (color online) (a) and (b) are the schematic diagrams for the chemical potential along a given boundary with (a) and without (b) spin dephasing. (c) and (d) plot the spin Hall resistance $R_{s}$ vs. $B_{\text {eff }}$. (e) and (f) illustrate the function $I_{2 s} / I_{14}$ vs. $B_{e f f}$. (c) and (e) are for the normal dephasing case, and (d) and (f) are for the spin dephase case. The parameters are $M=24 a, L=32 a$ and $W=32 a$.

than $R_{14,23}$. We find that a new spin Hall resistance $R_{s} \equiv\left(V_{i \uparrow}-V_{i \downarrow}\right) / I_{14}$ ( $i$ can be any transverse lead, for instance $i=2$ ) can fulfill the purpose. Figs.5c and $5 \mathrm{~d}$ show the spin Hall resistance $R_{s}$, and it exhibits the quantum plateaus at $h / 2 \nu e^{2}$ even with strong normal or spin dephasing. For example, at $\Gamma=0.1 t$, the sample length exceeds $L_{\phi}$ by one order of magnitude, the plateaus of $R_{s}$ still stay at the quantized values. This means that these plateaus will be visible in macroscopic QSHE samples. The robustness of $R_{s}$ against either dephasing is similar to what appeared in the Hall resistance plateaus in the conventional QHE (see Fig.1(b)). Let us explain the origin of the story with the aid of Figs.5a,b, in which the chemical potential along a given boundary is shown. In the left and right leads, the chemical potentials are always spin-independent with $V_{1 \sigma}=-V_{4 \sigma}=V$. In the central region, the chemical potential $V_{c \sigma}$ is spindependent. Without spin dephasing, $V_{c \uparrow}=V$ and $V_{c \downarrow}=-V$ (see Fig.5a) since the spin-up electrons flow to the right while the spin-down electrons to the left. In the presence of spin dephasing, the chemical potential $V_{c \sigma}$ descends along the longitudinal direction (see Fig.5b). But in order to keep the current $I_{14}$ to be a constant, $V_{c \uparrow}-V_{c \downarrow}$ needs to be also unchanged regardless of the positions along the sample and the spin dephasing strength $\Gamma$ since the current $I_{14}$ is carried by the edge states between $V_{c \uparrow}$ and $V_{c \downarrow}$. Therefore, the plateaus of the spin Hall resistance $R_{s}=\left(V_{2 \uparrow}-V_{2 \downarrow}\right) / I_{14}$ will stay unchanged even with strong spin-dephasing (i.e. in macroscopic samples). In addition, due to constant nature of $R_{s}$, the ratio of the transverse spin current to the longitudinal current(e.g.
$I_{2 s} / I_{14}$, see Fig.5e,f) and the spin accumulation on the boundary can also survive in the presence of strong normal and spin dephasings. Note that the spin accumulations and the difference of $V_{2 \uparrow}-V_{2 \downarrow}$ have been measured in recent experiments. 16, 17]

In summary, the effect of dephasing on QSHE is studied. We find that the quantum plateaus of the longitudinal resistance $R_{14,23}$ are insensitive to the normal dephasing, but are severely affected by the spin dephasing, so that these quantum plateaus exist only in mesoscopic samples. This result explains why the quantum plateaus of $R_{14,23}$ are only observed in small-size samples in the recent experiment. [8]. The dephasing effect also provides the understanding of observed dependence of $R_{14,23}$ on temperature, sample length and sample width. In addition, we find a new spin Hall resistance that also exhibits quantum plateaus. In particular, these plateaus stay at quantized values in macroscopic samples and better reflect the topological nature of QSHE.

This paper is supported by NSF-China under Grants Nos. 10525418 and 10734110, by US-DOE under Grants No. DE-FG02- 04ER46124 and US-NSF.

[*] Electronic address: sunqf@aphy.iphy.ac.cn

[1] For a brief review, see C. Day, Physics Today 6119 (2008); N. Nagaosa, Science 318, 758 (2007).

[2] C.L. Kane, E.J. Mele, Phys. Rev. Lett 95, 226801(2005).

[3] C.L. Kane, E.J. Mele, Phys. Rev. Lett 95, 146802(2005).

[4] B.A. Bernevig and S.-C. Zhang, Phys. Rev. Lett 96, 106802 (2006); X.-L. Qi, et al., Phys. Rev. B 74, 085308 (2006); C. Liu, et al., Phys. Rev. Lett 100, 236601 (2008).

[5] L. Fu, C.L. Kane, and E.J. Mele, Phys. Rev. Lett 98, 106803 (2007); L. Fu and C.L. Kane, Phys. Rev. B 76, 045302 (2007); J.C.Y. Teo, et al., Phys. Rev. B 78, 045426 (2008).

[6] L. Sheng, et al., Phys. Rev. Lett 95, 136602 (2005).

[7] B.A. Bernevig, T.L. Hughes and S.C. Zhang, Science 314, 1757 (2006).

[8] M. König, et al., Science 318, 766 (2007); M. König, et al., J. Phys. Soc. Jpn. 77, 031007 (2008).

[9] D. Hsieh, et al., Nature 452, 970 (2008).

[10] M. Büttiker, Phys. Rev. B 33, 3020 (1986); J.R. Shi and X.C. Xie, Phys. Rev. B 63, 045123 (2001).

[11] Y. Meir and N.S. Wingreen, Phys. Rev. Lett. 68, 2512 (1992); A.-P. Jauho, N.S. Wingreen, and Y. Meir, Phys. Rev. B 50, 5528 (1994).

[12] Electronic Transport in Mesoscopic Systems, edited by S. Datta (Cambridge University Press 1995).

[13] C. Wu, et al., Phys. Rev. Lett 96, 106401 (2006).

[14] Q.-F. Sun, et al., Phys. Rev. B 71, 165310 (2005).

[15] Y.X Xing, et al., Phys. Rev. B 77, 115346 (2008).

[16] E.J. Koop, et al., Phys. Rev. Lett. 101, 056602 (2008); Q.-F. Sun, et al., Phys. Rev. B 77, 195313 (2008); Y.X. Xing, et al., Appl. Phys. Lett. 93, 142107 (2008).

[17] Y.K. Kato, et al., Science 306, 1910 (2004); V. Sih, et al., Phys. Rev. Lett. 97, 096605 (2006). 